Mon. Not. R. Astron. Soc. 000, 000-000 (0000) Printed 16 June $2018 \quad$ (MN LATEX style file v2.2)

\title{
Fast radiative transfer of dust reprocessing in semi-analytic models with artificial neural networks
}

\author{
Laura Silva $^{1 \star}$, Fabio Fontanot ${ }^{2,3} \dagger$, Gian Luigi Granato ${ }^{1} \ddagger$ \\ 1 INAF-Trieste, Via G.B. Tiepolo 11, I-34131 Trieste, Italy \\ 2 HITS Heidelberger Institut für Theoretische Studien, Schloss-Wolfsbrunnenweg 35, 69118 Heidelberg, Germany \\ 3 Institut für Theoretische Physik, Philosophenweg, 16, 69120, Heidelberg, Germany
}

'Accepted ... Received ...

\begin{abstract}
A serious concern for semi-analytical galaxy formation models, aiming to simulate multi-wavelength surveys and to thoroughly explore the model parameter space, is the extremely time consuming numerical solution of the radiative transfer of stellar radiation through dusty media. To overcome this problem, we have implemented an artificial neural network algorithm in the radiative transfer code GRASIL, in order to significantly speed up the computation of the infrared SED. The ANN we have implemented is of general use, in that its input neurons are defined as those quantities effectively determining the shape of the IR SED. Therefore, the training of the ANN can be performed with any model and then applied to other models. We made a blind test to check the algorithm, by applying a net trained with a standard chemical evolution model (i.e. CHE_EVO) to a mock catalogue extracted from the SAM MORGANA, and compared galaxy counts and evolution of the luminosity functions in several nearIR to sub-mm bands, and also the spectral differences for a large subset of randomly extracted models. The ANN is able to excellently approximate the full computation, but with a gain in CPU time by $\sim 2$ orders of magnitude. It is only advisable that the training covers reasonably well the range of values of the input neurons in the application. Indeed in the sub-mm at high redshift, a tiny fraction of models with some sensible input neurons out of the range of the trained net cause wrong answer by the ANN. These are extreme starbursting models with high optical depths, favorably selected by sub-mm observations, and difficult to predict a priori.
\end{abstract}

Key words: radiative transfer - method: numerical - galaxies: evolution - infrared: galaxies

\section{INTRODUCTION}

The great observational advances of the last decades have demonstrated the ubiquitous presence of dust in galaxies, and its strong impact on their Spectral Energy Distribution (SED). Dust grains efficiently absorb and scatter radiation at short wavelengths $(\lambda<1 \mu m)$. The absorbed energy is thermally re-emitted at longer wavelengths in the infrared (IR) region $(\lambda>1 \mu m)$. Since the early estimates based on the IRAS satellite, it has been clear that dust in the ISM shifts from the optical-UV to the IR region at least $\sim 30 \%$ of the total power emitted by primary sources (stars and AGNs) in nearby galaxies, and that this fraction increases

\footnotetext{
* Email: silva@oats.inaf.it

$\dagger$ Email: fabio.fontanot@h-its.org

‡ Email: granato@oats.inaf.it
}

with increasing galactic activity (e.g. Sanders \& Mirabel 1996).

More recent IR and sub-mm surveys have demonstrated that the importance of dust reprocessing increases at high redshift and have allowed to directly map the evolution of galaxies with cosmic time (e.g. ISO $15 \mu \mathrm{m}$, Elbaz et al. 1999, 2002; Gruppioni et al. 2002; SCUBA $850 \mu \mathrm{m}$, Hughes et al. 1998; Chapman et al. 2005; Mortier et al. 2005; Spitzer from from 3.6 to $160 \mu \mathrm{m}$, Le Floch et al. 2005, Perez-Gonzalez et al. 2005, Babbedge et al. 2006, Franceschini et al. 2006). The ongoing Herschel observational campaigns, with SPIRE at 250,350 and $500 \mu \mathrm{m}$, and PACS at 70, 110 and $170 \mu \mathrm{m}$ are making it possible to carry out the first large-area surveys in the almost unexplored far-IR range (e.g. H-ATLAS, Eales et al. 2010, and HERMES, Oliver et al. 2010).

Dust complicates significantly the interpretation of galactic SED, in that it introduces a dependence on the ge- 
ometry and on the intrinsic and uncertain dust properties, and calls for the solution of numerically expensive radiative transfer problems.

The study of galaxy formation and evolution has been receiving increasing interest also from the theoretical point of view. The modeling of galaxy formation and evolution in cosmological context involves many processes at very different scales, from hundreds of Mpc to much less than a pc. The widest range of observed galaxy properties have been analyzed using the so-called semi-analytic models (SAM; White \& Rees 1978; Lacey \& Silk 1991; White \& Frenk 1991), that consist in calculating the evolution of the baryon component using simple analytical approximations, involving several adjustable parameters, while the evolution of the dark matter is calculated directly using Monte-Carlo techniques based on the extended Press-Schechter theory, Lagrangian perturbation theory or gravity-only N-body simulations (see e.g. Lacey \& Cole 1993; Monaco, Theuns \& Taffoni 2002; Springel et al. 2005).

The final step to compare the SAM predictions with observations, is the computation of the SED for each galaxy of mock galaxy catalogues, in a wavelength range as broad as possible. Indeed, given the strong uncertainties in the recipes adopted to describe the baryon physics, and the large set of free parameters, substantial progresses call for a complete multi-wavelength analysis of galaxy data. The full SED of model galaxies, at least in principle, should be calculated by appropriately taking into account for each galaxy its particular star formation and metallicity history and the geometrical information provided by the SAM. The simulated SED catalogue can then be compared to real observed galaxy surveys, so as to check whether the predictions are or are not representative of the real universe and to retrieve some information on the galaxy formation process.

Due to the complexity and the computational cost of taking into account dust reprocessing, most semi-analytical models have made use of simple empirical or semi-empirical treatments (e.g. Guiderdoni et al. 1998; Kauffmann et al. 1999; Somerville \& Primack 1999; Hatton et al. 2003; Blaizot et al. 2004; Kang et al. 2005; Kitzbichler \& White 2007). The only SAMs that includes a UV to sub-mm radiative transfer computed from first principle, exploiting self consistently all the information provided by the SAM, are GALFORM (Cole et al. 2000; Granato et al. 2000 [G00]; Baugh et al 2005; Lacey et al. 2008, 2010; Swinbank et al. 2008), MORGANA (MOdelling the Rise of GAlaxies aNd Active nuclei, Monaco et al. 2007; Fontanot et al. 2007, 2009a; Lo Faro et al. 2009; Fontanot \& Monaco 2010), and ABC (Anti-hierarchical Baryonic Collapse; Granato et al. 2004; Silva et al. 2005; Lapi et al. 2006). These models have been interfaced with GRASIL to make detailed comparisons and predictions in different spectral ranges.

GRASIL (Silva et al. 1998 [S98]; Silva 1999 [S99]; G00; Bressan, Silva \& Granato 2002; Panuzzo et al. 2003; Vega et al. 2005; Schurer et al. 2009) is a relatively realistic and flexible multi-wavelength model, which calculates a galactic SED in a reasonably short computing time, to be applied both to interpret observations and to make predictions in conjunction with galaxy formation models. To do this, it includes a sufficiently realistic bulge plus disk geometry, as well as the radiative transfer effects of different dusty environments and the clumping of stars and dust, but avoids exceedingly time consuming Monte Carlo calculations and allows for some degree of geometrical (axial and equatorial) symmetry. The model has been successfully applied in many contexts (e.g. Granato et al. 2004; Baugh et al. 2005; Silva et al. 2005; Panuzzo et al. 2007a,b; Iglesias-Paramo et al. 2007; Fontanot et al. 2007; Galliano et al. 2008; Vega et al. 2008; Lacey et al. 2008, 2010; Michalowski et al. 2009, 2010; Schurer et al. 2009; Santini et al. 2010).

In particular, GRASIL has been used quite extensively for calculating the SEDs for the above mentioned SAMs. However, the calculation of the IR SED by GRASIL is still the bottleneck of the whole process of running a realization of a SAM, limiting the usefulness of this popular approach to galaxy formation modeling.

To improve on this, we implemented in GRASIL the possibility of computing SEDs with an Artificial Neural Network (ANN) algorithm. In Silva et al. (2011; henceforth Paper I) we demonstrated that this approach can reduce the computing time by orders of magnitude, with very limited loss of accuracy. However, in Paper I the ANN was implemented only for the two simplest geometrical arrangements of GRASIL, pure disc or spherical distribution of stars and dust, and we tested and applied it to cases suited for these geometries. In particular, as a practical sample application in the field of galaxy formation models, we computed galaxy counts in the Herschel imaging bands for the ABC model for spheroidal galaxies. However these simplified geometries are insufficient for a fully fledged SAM. Therefore, in this paper, we present the implementation of the ANN for the mixed bulge+disc geometry. To validate the technique, we compare statistical properties of galaxies predicted by the well known SAM MORGANA (Monaco et al. 2007), as derived with full GRASIL computations and with the ANN method.

Almeida et al. (2010) have used the ANN algorithm to insert the GALFORM+GRASIL model into the Millennium Simulation, to study the properties of the population of sub$\mathrm{mm}$ galaxies. That method is substantially different from the one presented here. They identify the properties of the GALFORM galaxies which determine, through an ANN, their full GRASIL SEDs. The method is successful and extremely fast, since the ANN is used to compute the entire SED, not only the IR dust emission, as in our case. However their implementation is very specific to GALFORM, whose different realizations require each a a training of the ANN, and one for each output redshift.

The method we have implemented here is less fast but far more general, because the input is directly linked to the galactic properties effectively determining the portion of the SED dominated by dust emission (e.g optical depths, masses of dust, radiation field etc.), independently of any SAM. As a result, one single training is able to cover a large variety of applications, such as to explore the parameter space of a SAM, or to deal with different SAMs. Indeed, we stress that the ANN employed in this paper has not been trained with SEDs computed for the semi-analytic model MORGANA.

In Section 2 we recall the main properties of GRASIL; in Section 3 we provide some generalities on ANN, and in Section 3.1 we describe the implementation of ANN in GRASIL, the choice of the input neurons and the definition of the trained net; in Section 4 we describe the main characteristics of the SAM MORGANA, and in Section 5 we show some 
applications and examples. Finally our conclusions are presented in Section 6

\section{MODELLING SEDS WITH GRASIL}

\subsection{General description}

GRASIL (GRAphite \& SILicate) is a model meant to describe the far-UV to radio SED of galaxies, treating with particular care the effects of dust reprocessing on the stellar radiation. Its aim is to provide a relatively realistic and flexible modelling of galaxy SEDs, with a moderate computing time. Below we provide a summary of its features sufficient to understand our implementation of the ANN. We refer to the original papers for more details (in particular S98; S99; G00; Panuzzo et al. 2003; Vega et al. 2005). A somewhat more expanded summary can be found in Paper I.

- Galaxies are represented with stars and dust distributed in a bulge and/or a disc, adopting respectively a King and a double exponential profile (see e.g. Fig. 2.7 in S99 or Fig. 1 in G00 for a schematic representation of the geometry and components).

- Three different dusty environments and their corresponding interaction with stars are considered: the starforming molecular clouds (MCs) associated with newly-born stars, the diffuse medium ("cirrus") associated with more evolved stars, and the dusty envelopes around AGB stars (intermediate age stars), their relative contribution to the SED depending on the star formation history.

- The birth of stars within MCs and their gradual dispersion into the diffuse medium is accounted for by decreasing the fraction of energy stars emit within MCs with increasing age, over a typical "escape timescale" (Sec. 2.5 and Eq. 8 in S98 for more details). Therefore we account for the clumping of (young) stars and dust within a diffuse medium, and for a greater attenuation suffered by the youngest stars (agedependent attenuation)

- The dust model is made of graphite and silicate spherical grains with a continuous size distribution including grains in thermal equilibrium with the radiation field, very small grains fluctuating in temperature due to the absorption of single UV photons, and PAH molecules (optical properties by Draine \& Lee 1984; Laor \& Draine 1993; Li \& Draine 2001; Draine \& Li 2007). We compute the response to the incident radiation field for each type of grain.

- The RT is exactly solved for the MCs with the $\Lambda$ iteration algorithm (e.g. Granato \& Danese 1994). They are represented as spherically symmetric clouds. Star forming MCs typically have extremely high optical depths even in the IR, which means IR-produced photons are self-absorbed, thus requiring a full RT treatment.

- The model galaxy is binned in appropriately small volume elements. The radiation field is evaluated in each of them from the knowledge of the distribution of stars and dust. Consequently the local dust emission and the attenuated radiation along each desired line of sight is computed. The treatment of the RT and dust emission in the diffuse phase (the major bottleneck of the whole computation) is approximated, but adequate for most situations (see Section 2.5.2 in S98 and 2.5.3 in S99).

- Our reference library of SSPs is from Bressan et al.
(1998, 2002). We recall that the effects of the dusty envelopes around AGB stars and the radio emission (both thermal and non-thermal) are directly included in these SSPs. But any desired SSP library can be given in input to GRASIL (e.g. Fontanot \& Monaco 2010 tested the effects of both Bressan et al. and Maraston (2005) SSPs in MORGANA+GRASIL).

\subsection{Inputs}

The inputs required by GRASIL consist of the star formation, gas and metallicity evolution histories, and a set of geometrical parameters. The former ingredients can be provided by analytical star formation laws, or by "classical" chemical evolution models, or by more complex galaxy formation models (e.g. GALFORM, MORGANA, ABC, see the Introduction).

Here, as in Paper I, we have generated the star formation histories used to train the ANN with the code CHE_EVO (described in S99, see also Section 2.2 in Fontanot et al. $2009 \mathrm{~b})$. This implies that the training libraries are in principle different from the typical MORGANA SF histories. CHE_EVO computes the evolution of the SFR, mass of gas, metallicity, and of the chemical elements given an IMF and a SF law of the kind $S F R(t)=\nu M_{\text {gas }}(t)^{k}+f(t)$, where the first term is a Schmidt-type SF with efficiency $\nu$, and $f(t)$ is an analytical term. Note however that our approach is independent of this choice, and indeed our aim is to compute ANNs that can work with any engine to generate the SF histories.

The other inputs required by GRASIL, some of which may be provided by galaxy formation models, are:

- $f_{M C}$ : gas mass fraction in star forming MCs. It affects mainly the FIR-submm.

- $\tau_{M C}$ : optical depth of MCs. It affects strongly the midIR and the silicate absorption feature.

- $\mathrm{t}_{\text {esc }}$ : escape time scale of young stars from the MCs. It affects mainly the IR to UV-optical ratio.

- $\delta \equiv \mathrm{M}_{\text {dust }} / \mathrm{M}_{\text {gas }}$ : dust to gas mass ratio. It is customary to set it either to a fixed value or proportional to the metallicity, unless provided by a dust evolution model (e.g. as in Schurer et al. 2009).

- Bulge scale-radii (core radii of the King profile) and disk scale-radii and heights (of the double exponential) for stars and dust. The distribution of the radiation field relative to the dust determines the dust temperature distribution.

\section{COMPUTING SEDS WITH ARTIFICIAL NEURAL NETWORKS}

We refer the reader to Section 3 of Paper I for more details on the topic discussed in this section. In particular, their Section 3.1 provides a summary of basic concepts on ANNs most relevant for the present application.

In short, ANNs are computing algorithms resembling to some extent the behavior of brains. They consist of processing units (neurons in ANN language) with multiple connections to transmit a signal, organized as a network. These connections have adaptable strengths (synaptic weights) which modify the signal transmitted to and from each neuron. The training of the network is the process of adjusting the weights, so that the network learns to solve the specific problem at hand. 
In our specific case, we have demonstrated in Paper I that the network can be trained on a large set of precomputed SEDs to predict, with good accuracy, the response of the SED at each wavelength (output neurons) to variations of the physical parameters (input neurons).

\subsection{Implementing ANN in GRASIL}

Our strategy is to use an ANN to predict only the IR emission, both from molecular clouds as well as cirrus, which are the true bottlenecks of GRASIL computations. The direct calculation of the extinction of starlight by the molecular clouds and by the cirrus, which is much less demanding, provides the dust attenuated stellar radiation. Also, it yields the amount of energy absorbed by dust, and therefore the normalization for the two dust emission components, while the ANN predicts their spectral shapes. Since the CPU time required by the $\mathrm{ANN}$ is negligible with respect to the direct computation, we obtain orders of magnitude gains in the time performance.

Owing to the very different nature and treatment of the MCs and cirrus components, the quantities (input neurons) that determine their respective IR SEDs are different. Therefore we have implemented a distinct ANN for each of them, namely a standard feed-forward back-propagation MultiLayer Perceptron (MLP) with one hidden layer, using a sigmoid activation function from the input to the hidden layer (see Paper I for definitions). To create and use the trained net we have adapted and included in GRASIL the F90 code by B. Fiedler freely available at http://mensch.org/neural//

\subsection{Input and output neurons}

The identification of the input neurons is based on physical expectations corroborated by working experience with GRASIL. As such, they are closely related to, but not coincident with, GRASIL parameters. This is because different combinations of two or more parameters produce identical or very similar dust emission SEDs.

For instance the cirrus, and even more the MC dust emission, depends weakly on details of the spectral shape of the input stellar radiation, which means that different combinations of $\operatorname{SFR}(t), Z(t)$, galactic age $T_{\text {gal }}$, and $\mathrm{MC}$ escape timescale $t_{e s c}$ (which affects the fraction of starlight heating the $\mathrm{MC}$ and that heating the cirrus) may give rise to almost identical dust emission in one or both components.

The output neurons are the values of $\lambda L_{\lambda}$ in the IR region, both for molecular clouds and for the cirrus, which usually means several hundreds output neurons.

\section{Input neurons for molecular clouds}

The input neurons for MCs are the same as in Paper I, since the different geometry considered here does not affect the MC emission:

- $\tau_{M C} \propto \delta M_{M C} / R_{M C}^{2}$, the molecular cloud optical depth (conventionally given at $1 \mu \mathrm{m}$ ).

- $R_{M C}[p c] /\left(k \sqrt{(} L_{\star M C, 46}\right)$ the ratio of the molecular cloud radius over an estimate of the dust sublimation radius, i.e. the inner radius of the dust distribution. The latter depends on the luminosity of stars within each cloud (in $10^{46}$ $\mathrm{erg} / \mathrm{s})$. The constant $k$ depends on the adopted maximum temperature $T_{s}$ for dust, but its value is irrelevant as long as we use the same value when training and using the MLP.

\section{Input neurons for Cirrus}

In Paper I, to cope with the cirrus emission we needed a set of 6 input neurons for spherical symmetry and 9 for discs. For the mixed bulge and disc geometry considered here, 3 more neurons than the latter set are necessary. In the following list we used boldface to highlight points where there are differences or additions with respect to Paper I:

- $\log \left(L_{C i r} / L_{\star, c}\right)$, the cirrus dust luminosity normalized to the stellar luminosity heating the cirrus. The former is given by the amount of stellar plus MCs energy absorbed by the cirrus, from conservation of energy. This ratio provides a global measure of the amount of dust reprocessing.

- $\log \left(M_{C i r} / L_{\star, c}\right)$, the normalized cirrus dust mass, correlated with the (distribution of) dust emitting temperature.

- $\tau_{p}$ and $\tau_{e}$, the polar and equatorial optical depths due to cirrus alone (integral of the dust density distribution along the polar and equatorial directions respectively, conventionally given at $1 \mu \mathrm{m})$.

- $\tau_{h}$, a fictitious optical depth, computed as if the cirrus dust were spherically and homogeneously distributed. This dummy quantity measures the "concentration" of the dust distribution independently of the specific density law assumed. This concentration significantly affects the shape of the emitted SED, and we empirically found that its inclusion improves the performances of the MLP.

- Geometrical ratios: To the three ratios used in Paper I for the disc geometry, $r_{d, *} / r_{d, \text { diff } f}, z_{d, *} / r_{d, *}, z_{d, \text { diff } f} / r_{d, \text { diff }}$, we added the ratio $r_{d, *} / r_{c, *}$ between the scalelengths of the star distributions in the disk and bulge component. Taken together these four ratios characterize the relative position of dust and stars and the geometrical thickness of star and dust distributions.

- Hardness ratio: ratio of the radiation field at $0.3 \mu \mathrm{m}$ over $1 \mu \mathrm{m}$, heating the cirrus (thus emerging from molecular clouds and stars already out of molecular clouds). Since small grains and especially PAHs are excited most effectively by relatively hard UV photons, this quantity is correlated with the ratio between the NIR-MIR emission they produce, and the far-IR due to big grains.

- Bulge over total ratio $b / t$ : fraction of the stellar mass in the bulge component. This provides some information of the relative importance of the two stellar components in heating dust grains.

- Fraction of cirrus heating due to stars and not to MCs: $\log \left(1-\left(L_{M C}-L_{M C \text {,ext }}\right) / L_{C i r}\right)$, where $L_{M C}$ and $L_{M C \text {,ext }}$ are the emissions of MCs before and after absorbtion by the diffuse cirrus dust. This was not used in Paper I, though its usefulness does not depend on the particular geometry. The reason is that, in most real situations, the cirrus emission, peaking at $\sim 100 \mu \mathrm{m}$, is by far not selfabsorbed. Consistently, GRASIL does not deal with the radiative transfer of IR photons through this component. In this case even the MC emission, though peaking at shorter IR wavelength, where the optical depth is higher, is still negligibly absorbed by the cirrus. In this case all cirrus heating comes from starlight. However SAM can produce mock 
galaxies with thicker cirrus. In those cases in which the absorbtion of MC emission by cirrus is not negligible, but the cirrus self-absorbtion is still of little importance, GRASIL can still produce reliable SEDs. To cope with this with ANNs, we found empirically that it is useful to have an information on the fraction of cirrus heating coming from stars and MCs. This is likely due to the very different capability of typical starlight and MC photons to penetrate the diffuse cirrus distribution. In summary, the inclusion of this neuron improves the capability of GRASIL-ANN to work with extreme galaxies produced by SAMs.

\subsection{Network training}

The MLPs we use in the following have been trained on about $10^{4}$ CHE_EVO+GRASIL models, likely covering the range of parameter values arising in typical realizations of MORGANA:

- $\tau_{M C}=0.001$ to 50 ;

- $R_{M C} / R_{\text {min }}=160$ to 20000 ;

- $\log \left(L_{C i r} / L_{\star, c}\right)=-5$ to 0.1 ;

- $\log \left(M_{C i r} / L_{\star, c}\right)=-9.84$ to -5.84 ;

- $\tau_{e}=1.6 \mathrm{e}-5$ to 1500 ;

- $\tau_{p}=2.2 e-6$ to 95 ;

- $\tau_{h}=1.7 e-7$ to 1 ;

- $r_{d, *} / r_{d, \text { diff }}=0.5$ to 1 ;

- $z_{d, *} / r_{d, *}=0.05$ to 0.30 ;

- $z_{d, \text { diff } f} / r_{d, \text { diff }}=0.02$ to 0.3 ;

- $r_{d, *} / r_{c, *}=0.01$ to 100 ;

- $\log \left(L_{\star, c}(0.3) / L_{\star, c}(1)\right)=-0.46$ to 0.66 ;

- $b / t=0.02$ to 1 ;

- $\log \left(1-\left(L_{M C}-L_{M C, \text { ext }}\right) / L_{C i r}\right)=-0.34$ to 0 .

We trained the net with $90 \%$ of the models, randomly chosen within the library generated with the aforementioned range of parameters, and using the remaining $10 \%$ as a verification set. We have empirically adjusted the number of neurons in the hidden layer $n_{h i d}$ in the same way as in $\mathrm{Pa}-$ per I. We have adopted $n_{\text {hid }}=20$ for molecular clouds and 47 for cirrus. We trained the MLP using 750 training epochs (iterations) with a learning rate of 0.001 . We verified that none of these choices is very critical for the final results.

An important advantage of the ANN technique with respect to classical interpolations is the capability to "learn" the effect of each single input neuron on the SED, mimicking in some sense the skill that a real GRASIL user develops with experience. Therefore the MLP can approximate sufficiently well for most purposes the SED produced by a full GRASIL computation, corresponding to a given choice of input neurons, even when the trained set does not include examples with the entire set of inputs neurons bracketing the required ones at the same time. It is often sufficient that each single input neuron is independently within the values included for the training.

\subsection{Computing performance}

The implementation of the ANN into GRASIL dramatically reduces the $\mathrm{CPU}$ time required to run the code. In Fig. 11 we show the histogram of the CPU times required to compute the SED for our reference mock galaxy catalogue

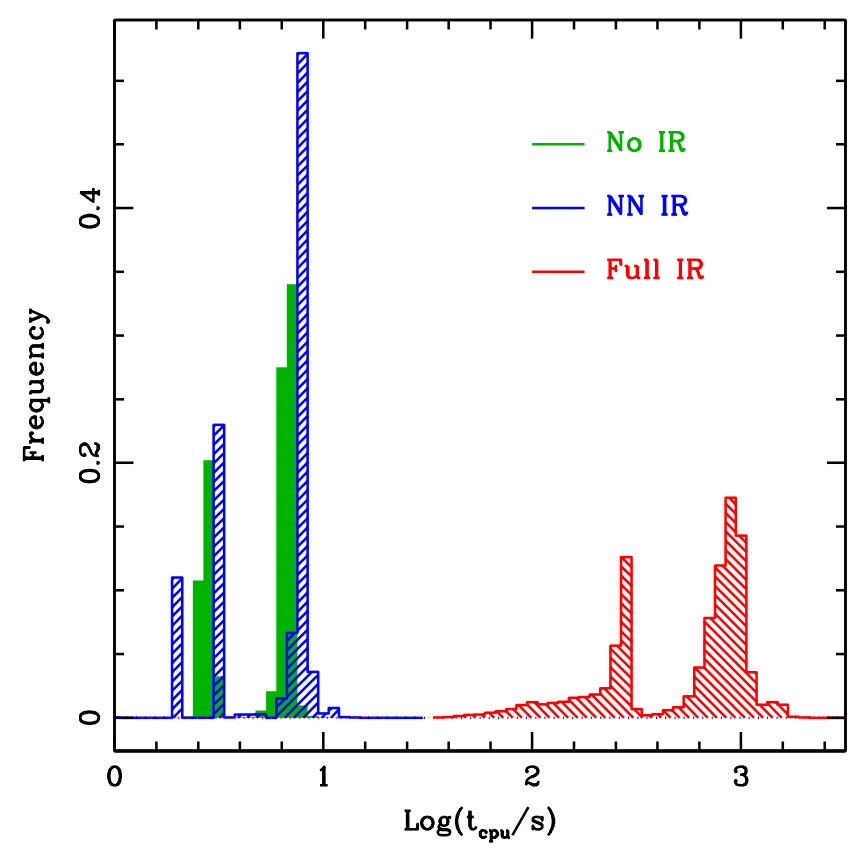

Figure 1. Comparison of the CPU computing time for the fulland the ANN-GRASIL in the case of a typical MORGANA realization. Backslash (red) histogram is for the full computation, the forward slash (blue) one is for the ANN, and the full (green) histogram is if only extinction is computed.

(see Section 5 for more details on the catalogue). The histograms show the computing time distributions for a run with only optical extinction (no IR computation, solid green histograms), a run with the full GRASIL computation (red backslash histograms), and with the ANN technique (blue forward slash histogram). The CPU gain between the full and the ANN computation is of about 2 orders of magnitude.

\section{SEMI-ANALYTIC MODEL: MORGANA}

To test the GRASIL-ANN, we make use of the semi-analytic model MORGANA, developed by Monaco et al. (2007) and Fontanot et al. (2007). We refer the reader to these papers for details on the galaxy formation model. Here we just recall its main features: the model implements a sophisticated treatment of mass and energy flows between the different gas phases (cold, hot and stars) and galactic components (bulge, disc and halo), as well as a new treatment for gas cooling and infall (following Viola et al. 2008). It also includes both a multi-phase description of star formation and feedback (following Monaco 2004) and a self consistent description of AGN activity and feedback (Fontanot et al. 2006). In the following we use the MORGANA realization updated to the WMAP3 cosmology and Chabrier IMF as in Lo Faro et al. (2009).

Every model galaxy is represented by assuming a composite geometry including both a spheroid and a disc component. Disc exponential profiles are computed following the Mo et al. (1998) formalism: the spin parameter of the DM halo is randomly extracted from a well defined distribution 


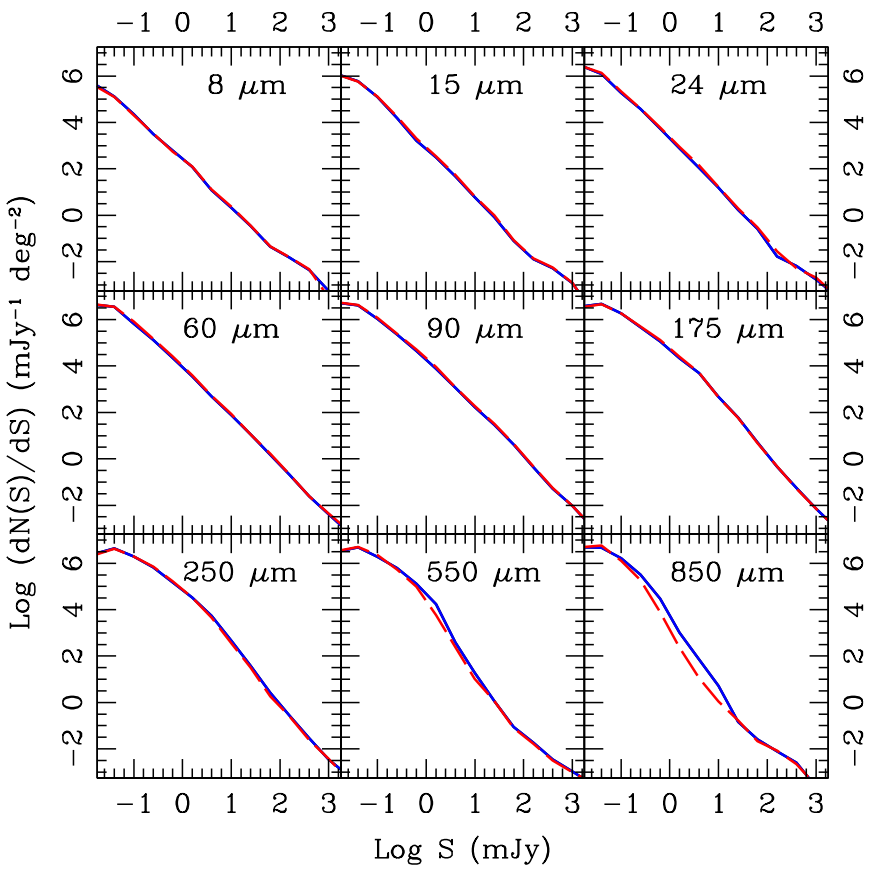

Figure 2. IR galaxy counts for MORGANA + GRASIL, comparing the full computation (dashed red line) and the ANN approximation (continuous blue line). The over-prediction in the ANN curve in the sub-mm is caused by a tiny fraction high redshift models with some input neurons out of the range of values covered by the trained net. See also Fig. 5 and text for explanation.

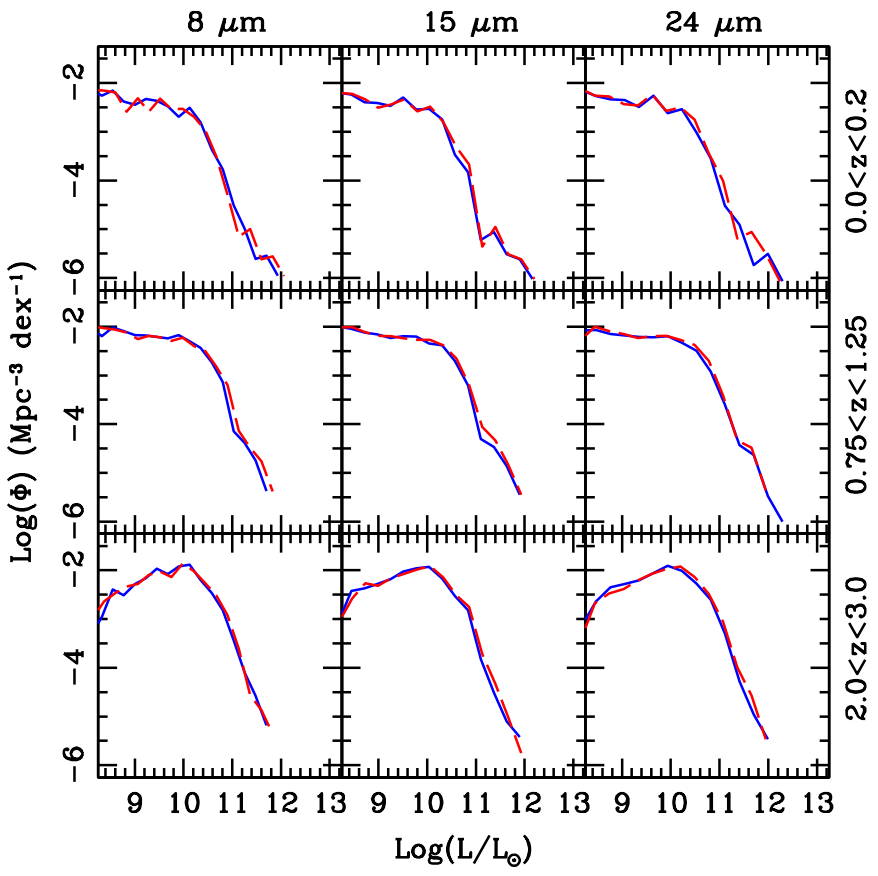

Figure 3. IR LF in the near and mid-IR bands and at three redshift bins for MORGANA +GRASIL, comparing the full computation (dashed red line) and the ANN approximation (continuous blue line).

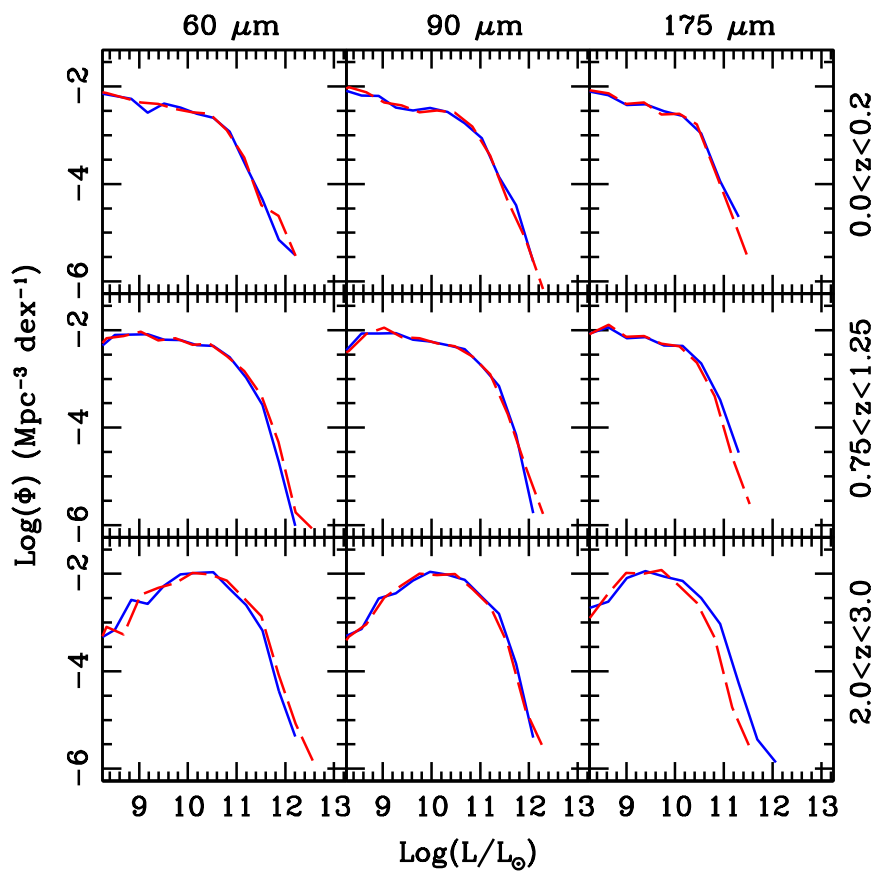

Figure 4. Same as Fig. 3 but in the FIR. The mismatch in the ANN curve at high redshift is due to models with some input neurons out of the range of values covered by the trained net. See text for explanation.

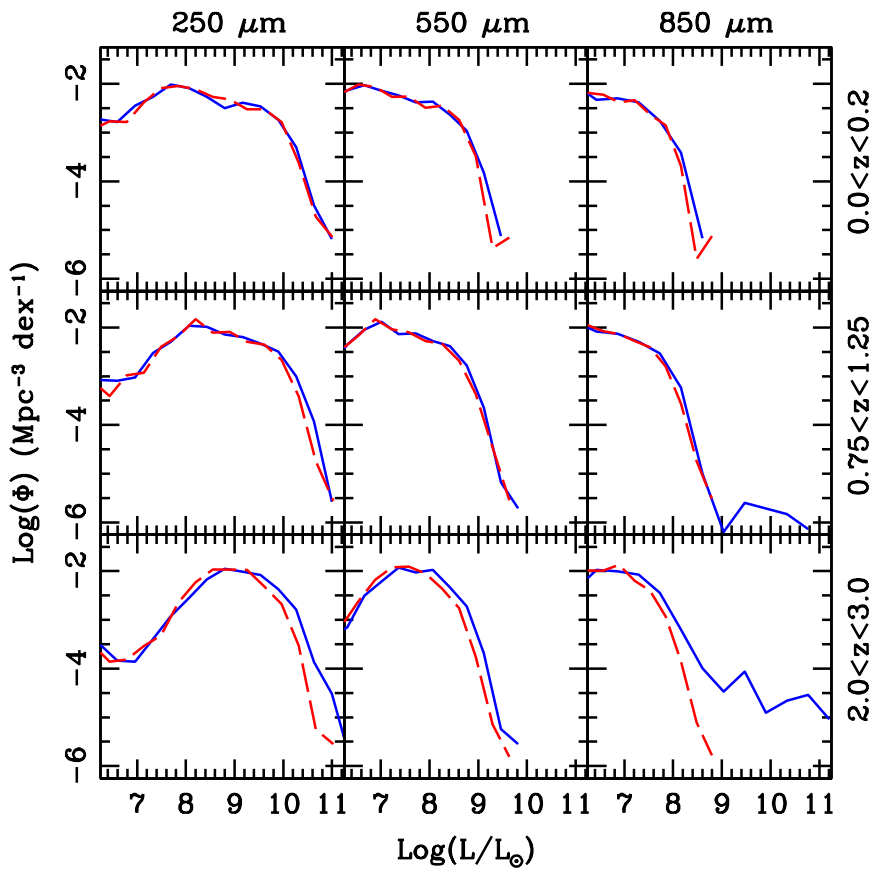

Figure 5. Same as Fig. 3 but in the FIR and sub-mm. The mismatch in the ANN curve at high redshift is due to models with some input neurons out of the range of values covered by the trained net. See text for explanation. 
and the angular momentum is conserved. Bulge sizes are computed assuming that the kinetic energy is conserved in merger events (Cole 2000). The presence of a bulge is taken into account when disc sizes are computed. MORGANA provides predictions for the star formation history, metal enrichment, and mass assembly of each component separately. This information is then interfaced with GRASIL in order to predict the resulting SED from the UV to the radio. The MORGANA realization we consider here is able to reproduce the local and $z=1$ stellar mass function, the cosmic star formation history, the evolution of the stellar mass density, the slope and normalization of the Tully-Fisher relation for spiral discs, the redshift distribution and luminosity function evolution for $K$-band selected samples, and, more interestingly, the number counts of $850 \mu \mathrm{m}$ selected sources. Despite these successes, the agreement between model predictions and observations of the apparent "downsized" trend of galaxy formation is still under debate (see Fontanot et al. 2007, 2009a for a complete discussion about "downsizing" trends and SAMs). It is well established that this model overpredicts the number of faint, low-mass galaxies at $z<2$ (Fontana et al. 2006; Fontanot et al. 2009a) and the space density of bright galaxies at $z<1$ (Monaco et al. 2006). Moreover, it does not reproduce the observed levels of star formation activity as a function of stellar and halo mass in the local universe (Kimm et al. 2009). Despite being a particular choice among the various SAM codes available in the literature, MORGANA is well suited for the purposes of this paper, since it includes all the relevant physical processes and it is representative of this technique (i.e. its predictions are consistent with other codes, see e.g. Fontanot et al. 2009a).

\section{RESULTS}

As recalled in the Introduction, recent IR and sub-mm observations have allowed to directly map the evolution of galaxies with cosmic time over large cosmological volumes. In order to exploit this huge amount of multi-wavelength data, we should be able to build large simulated volumes of galaxies and to assign to each of them its SED. Another fundamental application is a thorough exploration the parameter space of the SAM, practically unfeasible with the full GRASIL computation.

We recall that the input for the trained net is computed with CHE_EVO and that we have blindly applied this net to MORGANA. We run both the full and ANN versions of GRASIL on the same representative subsample of galaxies extracted from a MORGANA realization of a cosmological box of $200 \mathrm{Mpc}$ size, following the same sampling procedure described in Fontanot et al. (2007). Our final mock catalogue contains 95910 galaxies in a redshift range $0 \leqslant z \leqslant 8$. We have then extracted the statistical quantities, counts and LFs, from these two lightcones, and compared them (Figs. 2 to 5). This is done in several bands covering the whole nearIR to sub-mm range. Since in this paper we are interested in only assessing the validity of the ANN method in computing SEDs for SAMs, the comparison of this SAM with the data in IR bands, and the ensuing tuning of parameters and/or physical ingredients, will be investigated in another paper. In this work, we keep the same parameter values as defined in Lo Faro et al. (2009). Even if we found that $\sim 50 \%$ of mock galaxies have at least one input neuron out of the range of the trained net, only high-z starburst models with extreme values of the optical depth caused prediction of the SED by the ANN so bad to significantly affect sub-mm LFs and number counts. This is shown below.

Number counts are the most integrated observables used to test SAMs. Therefore spectral differences between the two computing methods for individual galaxies are smoothed out the most. In Fig. 2 we compare the MORGANA+GRASIL galaxy counts for the full (dashed red line) and the ANN (continuous blue line) computation in several bands: the Spitzer-IRAC band at $8 \mu \mathrm{m}$, the ISO 15, 90 and $175 \mu \mathrm{m}$ bans, the Spitzer-MIPS band at $24 \mu \mathrm{m}$, the IRAS $60 \mu \mathrm{m}$, the Herschel-SPIRE bands at 250 and $550 \mu \mathrm{m}$, and the $S C U B A 850 \mu \mathrm{m}$ band. The two curves are almost superimposed at all wavelengths except in sub-mm bands, at 550 and even more at $850 \mu \mathrm{m}$.

In order to further check our method, in Figs. 3, 4 and 5 we compare the ANN vs the full computation for the redshift evolution of MORGANA luminosity functions, at the same near- to far-IR and sub-mm bands as for the counts, in three redshift bins, $z=0-0.2,0.75-1.25,2-3$. Although this statistic is less integrated, we find again excellent agreement in most cases, but at high redshift and long wavelengths, due to those same models selected by the sub-mm counts.

We have verified that the over-prediction in the ANN curve at $850 \mu \mathrm{m}$ is due to $\sim 60$ objects (60 over 9678 at $z \sim 2.5$, and 5 over 17230 at $z \sim 1$ ) characterized mainly by extreme values of the luminosity and of the dust optical depth: for these models, in particular, the latter is far greater than the maximum values currently available in the trained net used here. More specifically, all these galaxies have a $1 \mu \mathrm{m}$ optical depth $\tau_{p}>100$ (the maximum value of this neuron in the trained net is 95), and actually most of them have $\tau_{p}$ between 200 and 300. Another characteristic for most of these models is an extremely high normalized cirrus luminosity above the maximum currently available $\left(\log \left(L_{C i r} / L_{\star, c}\right)=0.1\right)$. For these models the ANN provides an excess FIR-summ cirrus SED for $\tau_{p} \sim 100$, and a catastrophic answer for greater values. We explicitly note that these badly out-of-range models do not affect other spectral ranges. Indeed, as in the observations, sub-mm bands are particularly effective in selecting rare high-z, very dusty and very luminous extreme mock galaxies, due to their favorable negative k-corrections.

To investigate this point, we have randomly extracted from the MORGANA catalogue a sample of $10^{4}$ models, over the 0 to 8 redshift range and compared directly the spectral behavior of the ANN vs the full computation. In Figs. [6] and 7 we show the logarithmic ratios of the ANN to original MORGANA + GRASIL monochromatic luminosities versus the latter, at different wavelengths from 8 to $850 \mu \mathrm{m}$. The dots are the models (black for models with all the neurons within the range of the trained net, cyan for those models with at least one neuron out range). The line with asterisks and the short-dashed lines are respectively the median and the $0.1-0.9$ percentiles for in-range models. The same quantities for out-of-range models are the line with diamonds and the dot-dashed lines. The statistical noise appearing in some plots at low luminosities are due to having a few points falling within the luminosity bins used to evaluate the median and the percentiles. The rms log- 

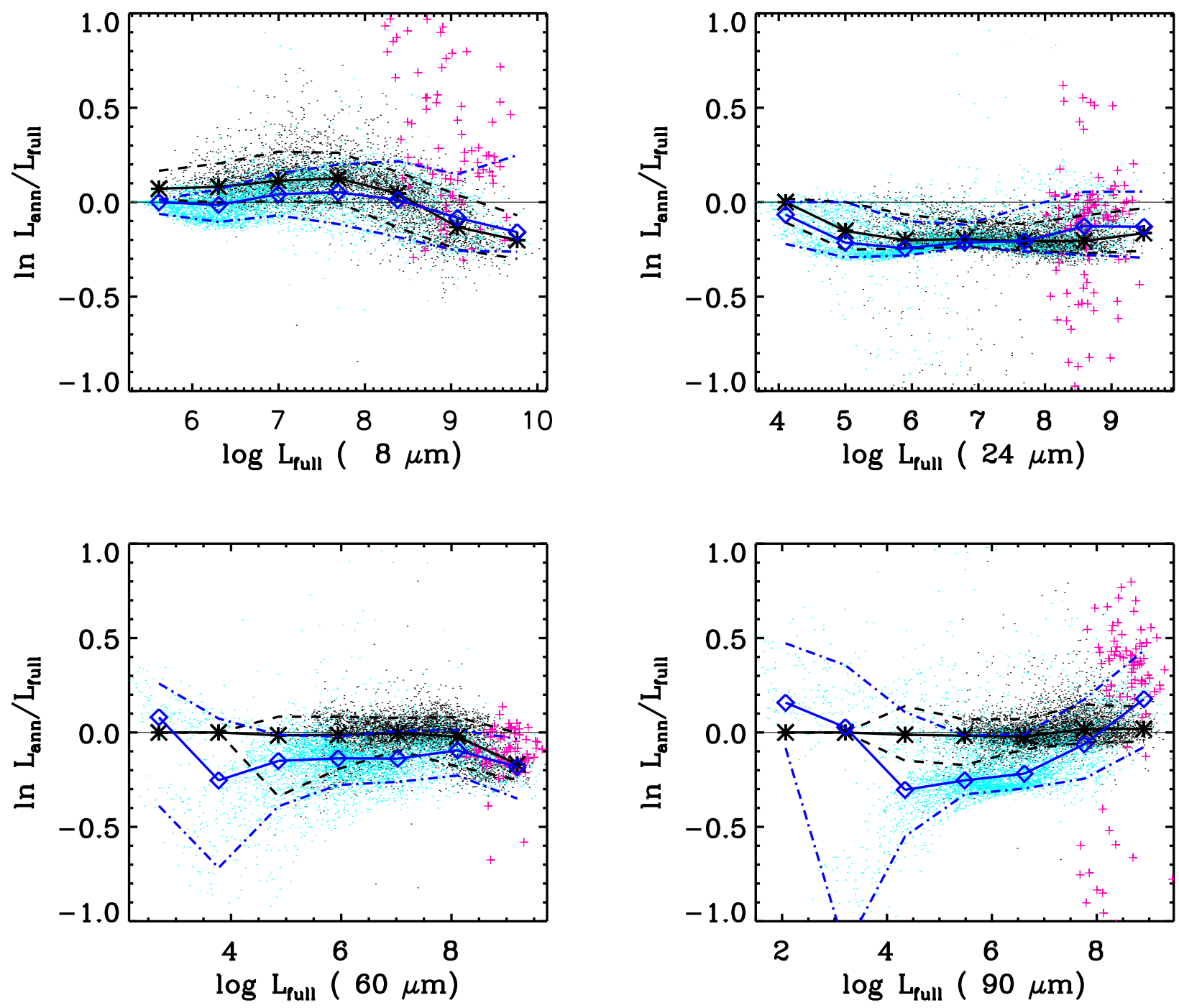

Figure 6. Logarithmic ratios of the ANN to the original monochromatic luminosities as a function of the latter (in $1 e 30$ erg/s/A), at 8, 24, 60, $90 \mu \mathrm{m}$ for $10^{4}$ randomly extracted MORGANA models. The black continuous line with asterisks and the short dashed lines are the median and the $0.1-0.9$ percentiles for those models (the black dots) having all their input neurons within the range of the trained net. The blue continuous line with diamonds and the dot-dashed lines are the same quantities for those models (the cyan dots) having at least one neuron out of the range of the trained net. Among these, extreme models with a polar optical depth $\tau_{p}>100$ at $1 \mu \mathrm{m}$ are highlighted by pink crosses.

arithmic errors at the different wavelengths are in Table 1 $\left.\left(e_{r m s}=\sqrt{(} \sum^{n}\left(\ln L_{a n n} / L_{f u l l}\right)^{2} / n\right)\right)$. There is a general dependency of the dispersion on the luminosity. In particular, the dispersion of the out-of-range models at the highest luminosity bins is dominated by extremely dusty models. In fact we have highlighted with crosses the models with a polar $1 \mu \mathrm{m}$ optical depth $\tau_{p}>100$. As we found previously for the sub-mm counts and LFs, also among this random sample, the models having a catastrophic answer by the ANN are all characterized by extreme values of $\tau_{p}$. In order to safely use (far-)IR data to constrain the SAM, it will be therefore advisable to enlarge the range of values covered by the training so to encompass also those extreme models predicted by the SAM but difficult to expect a priori.

\section{SUMMARY AND CONCLUSIONS}

It is well known that the numerical solution of the radiative transfer is time expensive, and this is particularly a concern for semi-analytical galaxy formation models, whose aim is to simulate large cosmological volumes to compare with multiwavelength surveys, or to thoroughly explore and constrain the model parameter space. On the other hand, the typical way to circumvent the computing time problem has been to rely on template SEDs. This can be misleading, since the templates, typically observationally based, can become very different from those that mock galaxies would have given their features, as predicted by the SAM (geometry and SF history).

We have implemented in GRASIL an artificial neural net- 

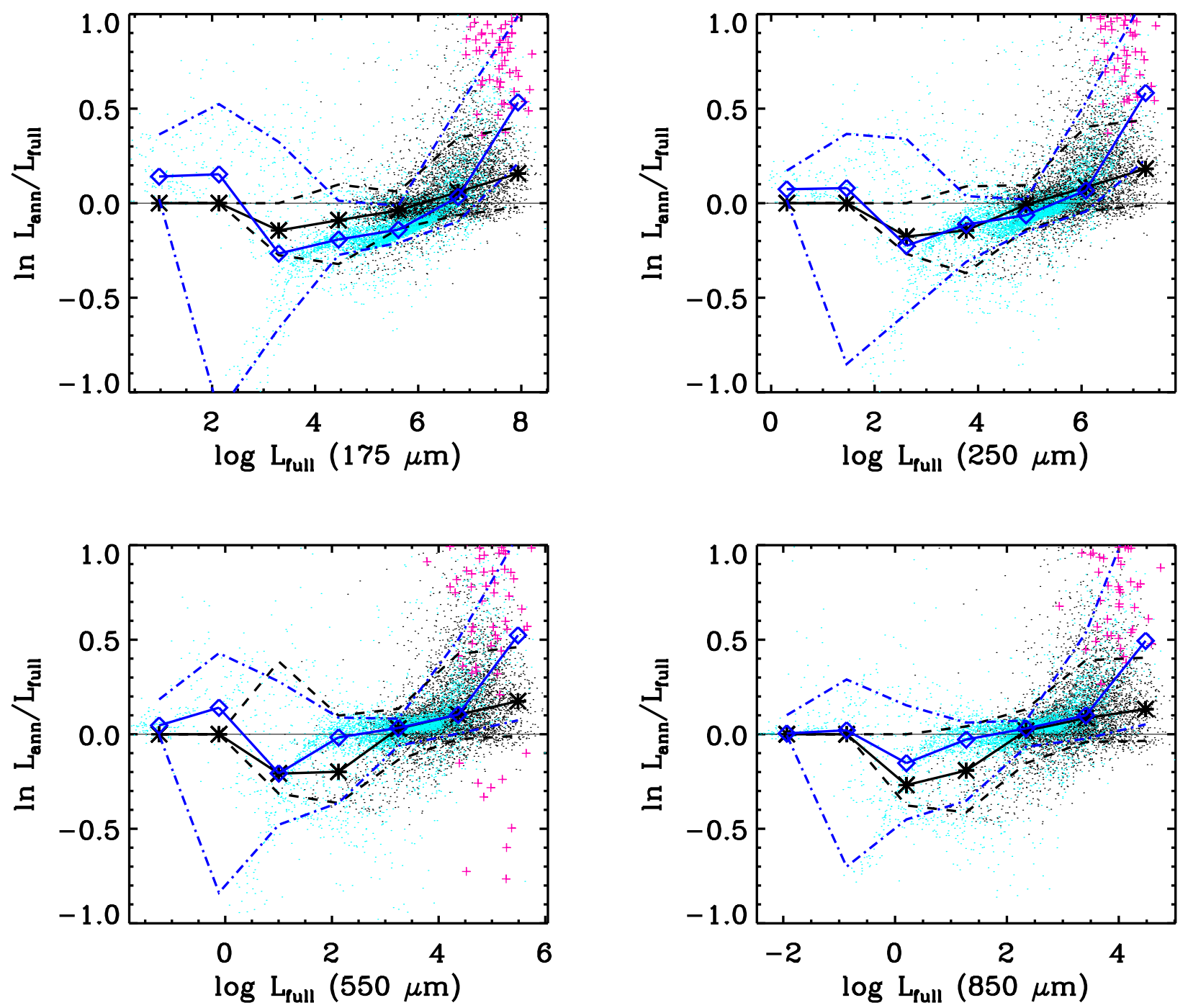

Figure 7. Same as Fig. 6 but at 175, 250, 550, $850 \mu \mathrm{m}$.

work algorithm in order to significantly speed up the computation of the IR SED, which is bottle-neck of the full SED computation. The ANN is able to excellently approximate the full computation, but with a gain in CPU time by $\sim 2$ orders of magnitude.

The main points of our work are as follows:

- In Paper I we have proven that the ANN algorithm was suited in the cases of the simplified pure bulge or pure disk geometries. Here we have further extended the ANN to the mixed bluge+disk geometry, suited for fully fledged SAMs. This required to account for additional input neurons to fully define the IR SEDs.

- An important characteristics of the ANN we have implemented is that it is of general use, i.e. it is not linked to a particular SAM. Indeed, the input neurons are defined as those quantities effectively determining the shape of the SEDs from the star forming clouds and the diffuse medium. Therefore, the training of the ANN can be performed with any model and then applied to other models. It is only ad- visable to cover the range of values of the input neurons encompassed by the model the net is applied to.

- We have checked the algorithm with the SAM MORGANA. We made a blind test, by applying a net trained with CHE_EVO (a standard chemical evolution model) covering a large range of input values, to a MORGANA catalogue. We ran the full and the ANN GRASIL on this catalogue, and compared the results for the galaxy counts and evolution of the LFs in several near-IR to sub-mm bands. The agreement is excellent except in the sub-mm at high redshift. We have checked that the disagreement at long wavelength is caused by a tiny fraction of models with some sensible input neurons out of the range of the trained net. These are extreme starbursting models with very high optical depths, favorably selected by sub-mm observations.

- Since counts, and to a lesser extent LFs, are integrated quantities for which small spectral differences are smoothed out, we have also performed a check on the spectral differences between the full and the ANN, by randomly selecting 
Table 1. RMS logarithmic error of the spectra predicted by the ANN with respect to the full computation at the wavelengths of Figs. 6] and 7 for a sample of $10^{4}$ randomly extracted models. The errors are given for the models with all neurons within the trained net, for those with at least one neuron out, and for the whole sample.

\begin{tabular}{cccc}
\hline$\lambda[\mu \mathrm{m}]$ & $e_{r m s}($ in $)$ & $e_{r m s}($ out $)$ & $e_{r m s}($ all $)$ \\
\hline 8 & 0.157 & 0.175 & 0.167 \\
24 & 0.229 & 0.255 & 0.243 \\
60 & 0.107 & 0.268 & 0.205 \\
90 & 0.104 & 0.311 & 0.233 \\
175 & 0.202 & 0.347 & 0.285 \\
250 & 0.235 & 0.350 & 0.298 \\
550 & 0.257 & 0.310 & 0.285 \\
850 & 0.240 & 0.533 & 0.414 \\
\hline
\end{tabular}

$10^{4}$ models from the MORGANA catalogue. We compare the median and the percentiles of the spectral luminosity ratios $L_{A N N} / L_{f u l l}$, separately for those models having all the neurons within the range of the trained net, and those with at least one neuron out. We confirm the previous findings for the goodness of the algorithm, and also that the large spread of out-of-range models take place at high luminosities and is ascribed to extremely dusty galaxies produced by MORGANA at high-z.

We conclude that the performance of the ANN algorithm is perfectly suited for the purpose of simulating galaxy lightcones with SAM and exploiting data from multiwavelength cosmological surveys, and for effective exploration of SAMs parameter space. The fact that this implementation is independent of the specific model used for generating the training set, will allow a single large training to be applied to different SAMs. The results are totally safe, at least for statistical purposes such as LFs and counts, provided that the training covers reasonably well the range of input neurons in the application. This condition is easy to check. In the event it were not satisfied, possible solutions are to broaden the training, or to run the full GRASIL for a few problematic mock galaxies. The best choice depends on the frequency of the latter and on the desired application. In this work we have used MORGANA as a realistic test for the ANN method. The comparison of MORGANA with IR data, and the likely tuning of its parameters and/or physical ingredients, exploiting this technique, will be the subject of a future work.

\section{ACKNOWLEDGMENTS}

FF acknowledges financial support from the Klaus Tschira Foundation. Some of the calculations were carried out on the "Magny" cluster of the Heidelberger Institute für Theoretische Studien.

\section{REFERENCES}

Almeida, C., Baugh, C. M., Lacey, C. G., Frenk, C. S., Granato, G. L., Silva, L., Bressan, A., 2010, MNRAS, 402, 544
Babbedge, T. S. R., Rowan-Robinson, M., Vaccari, M., Surace, J. A., Lonsdale, C. J., Clements, D. L., Fang, F., Farrah, D., et al., 2006, MNRAS, 370, 1159

Baugh C.M., Lacey C.G., Frenk C.S., Granato G.L., Silva L., Bressan A., Benson A.J., Cole S., 2005, MNRAS, 356 ,1191

Blaizot, J., Guiderdoni, B., Devriendt, J. E. G., Bouchet, F. R., Hatton, S. J., Stoehr, F., 2004, MNRAS, 352, 571

Bressan, A., Granato, G. L., Silva, L., 1998, A\&A, 332, 135

Bressan A., Silva L., Granato G.L., 2002, A\&A, 392, 377

Chapman, S. C., Blain, A. W., Smail, I., Ivison, R. J., 2005, ApJ, 622,772

Cole, S., Lacey, C. G., Baugh, C. M., Frenk, C. S., 2000, MNRAS, 319,168

Draine, B. T., Lee, H. M., 1984, ApJ, 285, 89

Draine, B. T., Li, A., 2007, ApJ, 657, 810

Eales, S., Dunne, L., Clements, D., Cooray, A., de Zotti, G., Dye, S., Ivison, R., Jarvis, M., et al., 2010, PASP, 122, 499

Elbaz, D., Cesarsky, C. J., Fadda, D., Aussel, H., Dsert, F. X., Franceschini, A., Flores, H., Harwit, M., et al., 1999, A\&A, 351, 37

Elbaz, D., Cesarsky, C. J., Chanial, P., Aussel, H., Franceschini, A., Fadda, D., Chary, R. R., 2002, A\&A, 384, 848

Fontana, A., Salimbeni, S., Grazian, A., Giallongo, E., Pentericci, L., Nonino, M., Fontanot, F., Menci, N., et al., 2006, A\&A, 459,745

Fontanot, F., Monaco, P., Cristiani, S., Tozzi, P., 2006, MNRAS, 373,1173

Fontanot, F., Monaco, P., Silva, L., Grazian, A., 2007, MNRAS, 382,903

Fontanot, F., De Lucia, G., Monaco, P., Somerville, R. S., Santini, P., 2009a, MNRAS, 397, 1776

Fontanot, F., Somerville, R.S., Silva, L., Monaco, P., Skibba, R., 2009b, MNRAS, 392, 553

Fontanot, F., Monaco, P., 2010, MNRAS, 405, 705

Franceschini, A., Rodighiero, G., Cassata, P., Berta, S., Vaccari, M., Nonino, M., Vanzella, E., Hatziminaoglou, E., et al., 2006, A\&A, 453, 397

Galliano, E., Alloin, D., Pantin, E., Granato, G. L., Delva, P., Silva, L., Lagage, P. O., Panuzzo, P., 2008, A\&A, 492, 3

Granato, G. L., Danese, L., 1994, MNRAS, 268, 235

Granato, G. L., Lacey, C. G., Silva, L., Bressan, A., Baugh, C. M., Cole, S., Frenk, C. S., 2000, ApJ, 542, 710

Granato, G. L., De Zotti, G., Silva, L., Bressan, A., Danese, L., 2004, ApJ, 600, 580

Gruppioni, C., Lari, C., Pozzi, F., Zamorani, G., Franceschini, A., Oliver, S., Rowan-Robinson, M., Serjeant, S., 2002, MNRAS, 335,831

Guiderdoni, B., Hivon, E., Bouchet, F. R., Maffei, B., 1998, MNRAS, 295, 877

Hatton, S., Devriendt, J. E. G., Ninin, S., Bouchet, F. R., Guiderdoni, B., Vibert, D., 2003, MNRAS, 343, 75

Hughes, D.H., Serjeant, S., Dunlop, J., Rowan-Robinson, M., Blain, A., Mann, R.G., Ivison, R., Peacock, J., 1998, Nature, 394, 241

Iglesias-Pramo, J., Buat, V., Hernndez-Fernndez, J., Xu, C. K., Burgarella, D., Takeuchi, T. T., Boselli, A., Shupe, D., et al., 2007, ApJ, 670, 279

Kang, X., Jing, Y. P., Mo, H. J., Brner, G., 2005, ApJ, 631, 21

Kauffmann, G., Colberg, J. M., Diaferio, A., White, S. D. M., 1999, MNRAS, 303, 188

Kimm, T., Somerville, R. S., Yi, S. K., van den Bosch, F. C., Salim, S., Fontanot, F., Monaco, P., Mo, H., 2009, MNRAS, 394, 1131

Kitzbichler, M. G., White, S. D. M., 2007, MNRAS, 376, 2

Lacey, C., Silk, J., 1991, ApJ, 381, 14

Lacey, C., Cole, S., 1993, MNRAS, 262, 627

Lacey, C. G., Baugh, C. M., Frenk, C. S., Silva, L., Granato, G. L., Bressan, A., 2008, MNRAS, 385, 1155 
Lacey, C. G., Baugh, C. M., Frenk, C. S., Benson, A. J., Orsi, A., Silva, L., Granato, G. L., Bressan, A., 2010, MNRAS, 405, 2 Laor, A., Draine, B. T., 1993, ApJ, 402, 441

Lapi, A., Shankar, F., Mao, J., Granato, G. L., Silva, L., De Zotti, G., Danese, L., 2006, ApJ, 650, 42

Le Floc'h, E., Papovich, C., Dole, H., Bell, E. F., Lagache, G., Rieke, G. H., Egami, E., Prez-Gonzlez, P. G., et al., 2005, ApJ, 632, 169

Li, A., Draine, B. T., 2001, ApJ, 554, 778

Lo Faro, B., Monaco, P., Vanzella, E., Fontanot, F., Silva, L., Cristiani, S., 2009, MNRAS, 399, 827

Maraston, C., 2005, MNRAS, 362, 799

Michalowski, M.J., Hjorth, J., Malesani, D., Michalowski, T., Castro Cern, J.M., Reinfrank, R.F., Garrett, M.A., Fynbo, J. P. U., Watson, D. J., Jrgensen, U. G., 2009, ApJ, 693, 347

Michalowski, M.J., Watson, D., Hjorth, J., 2010, ApJ, 712, 942

Mo, H. J., Mao, S., White, S. D. M., 1998, MNRAS, 295, 319

Monaco, P., 2004, MNRAS, 352, 181

Monaco, P., Murante, G., Borgani, S., Fontanot, F., 2006, ApJL, 652,89

Monaco, P., Fontanot, F., Taffoni, G., 2007, MNRAS, 375, 1189

Mortier, A. M. J., Serjeant, S., Dunlop, J. S., Scott, S. E., Ade, P., Alexander, D., Almaini, O., Aretxaga, I., et al., 2005, MNRAS, 363, 563

Oliver, S. J., Wang, L., Smith, A. J., Altieri, B., Amblard, A., Arumugam, V., Auld, R., Aussel, H., et al., 2010, A\&A, 518, 21

Panuzzo, P., Bressan, A., Granato, G. L., Silva, L., Danese, L., 2003, A\&A, 409, 99

Panuzzo, P., Granato, G. L., Buat, V., Inoue, A. K., Silva, L., Iglesias-Pramo, J., Bressan, A., 2007a, MNRAS, 375, 640

Panuzzo, P., Vega, O., Bressan, A., Buson, L., Clemens, M., Rampazzo, R., Silva, L., Valds, J. R., Granato, G. L., Danese, L., 2007b, ApJ, 656, 206

Prez-Gonzlez, P. G., Rieke, G. H., Egami, E., Alonso-Herrero, A., Dole, H., Papovich, C., Blaylock, M., Jones, J., et al., 2005, ApJ, 630, 82

Sanders, D. B., Mirabel, I. F., 1996, ARA\&A, 34, 749

Santini, P., Maiolino, R., Magnelli, B., Silva, L., Grazian, A., Altieri, B., Andreani, P., Aussel H., et al., 2010, A\&AL, 518, 154

Schurer, A., Calura, F., Silva, L., Pipino, A., Granato, G. L., Matteucci, F., Maiolino, R., 2009, MNRAS, 394, 2001

Silva L., Granato G.L., Bressan A., Danese L., 1998, ApJ, 509, 103 [S98]

Silva L., 1999, PhD thesis [S99]

Silva, L., De Zotti, G., Granato, G. L., Maiolino, R., Danese, L., 2005, MNRAS, 357, 1295

Silva, L., Schurer, A., Granato, G. L., Almeida, C., Baugh, C. M., Frenk, C. S., Lacey, C. G., Paoletti, L., et al., 2011, MNRAS, 410, 2043 (Paper I)

Somerville, R. S.; Primack, J. R., 1999, MNRAS, 310, 1087

Swinbank, A. M., Lacey, C. G., Smail, I., Baugh, C. M., Frenk, C. S., Blain, A. W., Chapman, S. C., Coppin, K. E. K., Ivison, R. J., Gonzalez, J. E., Hainline, L. J., 2008, MNRAS, 391, 420

Vega O., Silva L., Panuzzo P., Bressan A., Granato G. L., Chavez M., 2005, MNRAS, 364, 1286

Vega, O., Clemens, M. S., Bressan, A., Granato, G. L., Silva, L., Panuzzo, P., 2008, A\&A, 484, 631

Viola, M., Monaco, P., Borgani, S., Murante, G., Tornatore, L., 2008, MNRAS, 383, 777

White, S. D. M., Rees, M. J., 1978, MNRAS, 183, 341

White, S. D. M.; Frenk, C. S., 1991, ApJ, 379, 52 